\title{
Specify Other Preoperative IV Anesthesia Administered
}

National Cancer Institute

\section{Source}

National Cancer Institute. Specify Other Preoperative IV Anesthesia Administered. NCI

Thesaurus. Code C159585.

A request to specify intravenous anesthesia that was administered but not included on a list. 